The Journal of $\mathbf{N}_{\text {onlinear }} \mathbf{S}_{\text {ciences and }}$ Applications http://www.tjnsa.com

\title{
INTEGRAL MEANS OF ANALYTIC MAPPINGS BY ITERATION OF JANOWSKI FUNCTIONS
}

\author{
K. O. BABALOLA ${ }^{1}$ \\ Communicated by Prof. Ismat Beg
}

\begin{abstract}
In this short note we apply certain iteration of the Janowski functions to estimate the integral means of some analytic and univalent mappings of $|z|<1$. Our method of proof follows an earlier one due to Leung [4].
\end{abstract}

\section{IntRoduction}

Let $A$ be the class of normalized analytic functions $f(z)=z+a_{2} z^{2}+\ldots$ in the unit disk $|z|<1$. In $[2]$, among others, we added a new generalization class, namely; $T_{n}^{\alpha}[a, b], \alpha>0,-1 \leq b<a \leq 1$ and $n \in \mathbb{N}$; to the large body of analytic and univalent mappings of the unit disk $|z|<1$. This consists of functions in $|z|<1$ satisfying the geometric conditions

$$
\frac{D^{n} f(z)^{\alpha}}{\alpha^{n} z^{\alpha}} \in P[a, b]
$$

where $P[a, b]$ is the family of Janowski functions $p(z)=1+c_{1} z+\cdots$ which are subordinate to $L_{0}(a, b: z)=(1+a z) /(1+b z),-1 \leq b<a \leq 1$, in $|z|<1$. The operator $D^{n}$, defined as $D^{n} f(z)=z\left[D^{n-1)} f(z)\right]^{\prime}$ with $D^{0} f(z)=f(z)$, is the well known Salagean derivative [5].

In Section 2 of the paper [2] we extended certain integral iteration of the class of Caratheodory functions (which we developed in [1]) to $P[a, b]$ via which the new class, $T_{n}^{\alpha}[a, b]$, was studied. The extension was obtained simply by choosing

Date: Revised: 29 Sep. 2010.

(C) 2010 N.A.G.

2000 Mathematics Subject Classification. 30C45.

Key words and phrases. Integral means, iteration technique, analytic and univalent functions, Janowski functions. 
the analytic function $p(z)=1+c_{1} z+\cdots, \operatorname{Re} p(z)>0$ from $P[a, b]$ in the iteration defined in [1] as:

with $p_{0}(z)=p(z)$.

$$
p_{n}(z)=\frac{\alpha}{z^{\alpha}} \int_{0}^{z} t^{\alpha-1} p_{n-1}(t) d t, \quad n \geq 1
$$

We will denote this extention by $P_{n}[a, b]$ in this note. We had remarked (in [2]) that the statements (i) $p(z) \prec L_{0}(a, b: z)$, (ii) $p \in P[a, b]$, (iii) $p_{n}(z) \in P_{n}[a, b]$ and (iv) $p_{n} \prec L_{n}(a, b: z)$ are all equivalent. Thus we also remarked that (1) is equivalent to $f(z)^{\alpha} / z^{\alpha} \in P_{n}[a, b]$. This new equivalent geometric condition will lead us to the following interesting results regarding the integral means of functions in $T_{n}^{\alpha}[a, b]$ for $0<\alpha \leq 1$ and $n \geq 1$.

Theorem 1.1. Let $\Phi$ be a convex non-decreasing function $\Phi$ on $(-\infty, \infty)$. Then for $f \in T_{n}^{\alpha}[a, b], \alpha \in(0,1], n \geq 1$ and $r \in(0,1)$

$$
\int_{-\pi}^{\pi} \Phi\left(\log \left|f^{\prime}\left(r e^{i \theta}\right)\right|\right) d \theta \leq \int_{-\pi}^{\pi} \Phi\left(\log \left|\frac{L_{n-1}\left(a, b: r e^{i \theta}\right) k^{\prime}\left(r e^{i \theta}\right)^{1-\alpha}}{L_{0}\left(r e^{i \theta}\right)^{1-\alpha}}\right|\right) d \theta
$$

where

$$
L_{n}(a, b: z)=\frac{\alpha}{z^{\alpha}} \int_{0}^{z} t^{\alpha-1} L_{n-1}(a, b: t) d t, \quad n \geq 1
$$

and $k(z)=z /(1-z)^{2}$ is the Koebe function.

Theorem 1.2. With the same hypothesis as in Theorem 1, we have

$$
\int_{-\pi}^{\pi} \Phi\left(-\log \left|f^{\prime}\left(r e^{i \theta}\right)\right|\right) d \theta \leq \int_{-\pi}^{\pi} \Phi\left(-\log \left|\frac{L_{n-1}\left(a, b: r e^{i \theta}\right) k^{\prime}\left(r e^{i \theta}\right)^{1-\alpha}}{L_{0}\left(r e^{i \theta}\right)^{1-\alpha}}\right|\right) d \theta .
$$

The above inequalities represent the integral means of functions of the class $T_{n}^{\alpha}[a, b]$ for $\alpha \in(0,1]$ and $n \geq 1$. Our method of proof follows an earlier one due to Leung [4] using the equivalent geometric relations $f(z)^{\alpha} / z^{\alpha} \in P_{n}[a, b]$ for $f \in T_{n}^{\alpha}[a, b]$.

It is worthy of note that very many particular cases of the above results can be obtained by specifying the parameters $n, \alpha, a$ and $b$ as appropriate. In particular, the following special cases of $P[a, b]$ are well known: $P[1,-1] ; P[1-2 \beta,-1]$, $0 \leq \beta<1 ; P[1,1 / \beta-1], \beta>1 / 2 ; P[\beta,-\beta], 0<\beta \leq 1$ and $P[\beta, 0], 0<\beta \leq 1$ (see [2]). Thus several cases of $T_{n}^{\alpha}[a, b]$ may also be deduced.

\section{Fundamental Lemmas}

The following results are due to Baernstein [3] and Leung [4]. Let $g(x)$ be a realvalued integrable function on $[-\pi, \pi]$. Define $g^{*}(x)=\sup _{|E|=2 \theta} \int_{E} g,(0 \leq \theta \leq \pi)$ where $|E|$ denotes the Lebesgue measure of the set $E$ in $[-\pi, \pi]$. Further details can be found in the Baernstein's work [3].

Lemma $2.1([3])$. For $g, h \in L^{1}[-\pi, \pi]$, the following statements are equivalent:

(i) For every convex non-decreasing function $\Phi$ on $(-\infty, \infty)$,

$$
\int_{-\pi}^{\pi} \Phi(g(x)) d x \leq \int_{-\pi}^{\pi} \Phi(h(x)) d x .
$$


(ii) For every $t \in(-\infty, \infty)$,

$$
\int_{-\pi}^{\pi}[g(x)-t]^{+} d x \leq \int_{-\pi}^{\pi}[h(x)-t]^{+} d x .
$$

(iii) $g^{*}(\theta) \leq h^{*}(\theta),(0 \leq \theta \leq \pi)$.

Lemma $2.2([3])$. If $f$ is normalized and univalent in $|z|<1$, then for each $r \in(0,1),\left( \pm \log \mid f\left(r e^{i \theta} \mid\right)^{*} \leq\left( \pm \log \mid k\left(r e^{i \theta} \mid\right)^{*}\right.\right.$.

Lemma $2.3([4])$. For $g, h \in L^{1}[-\pi, \pi],[g(\theta)+h(\theta)]^{*} \leq g^{*}(\theta)+h^{*}(\theta)$. Equality holds if $g, h$ are both symmetric in $[-\pi, \pi]$ and nonincreasing in $[0, \pi]$.

Lemma 2.4 ([4]). If $g, h$ are subharmonic in $|z|<1$ and $g$ is subordinate to $h$, then for each $r \in(0,1), g^{*}\left(r e^{i \theta}\right) \leq h^{*}\left(r e^{i \theta}\right),(0 \leq \theta \leq \pi)$.

Corollary 2.5. If $p \in P_{n}[a, b]$, then

$$
\left( \pm \log \mid p_{n}\left(r e^{i \theta} \mid\right)^{*} \leq\left( \pm \log \mid L_{n}\left(a, b: r e^{i \theta} \mid\right)^{*}, \quad 0 \leq \theta \leq \pi .\right.\right.
$$

Proof. Since $p_{n}(z)$ and $L_{n}(a, b: z)$ are analytic, $\log \left|p_{n}(z)\right|$ and $\log \left|L_{n}(a, b: z)\right|$ are both subharmonic in $|z|<1$. Furthermore, since $p_{n} \prec L_{n}(a, b: z)$, there exists $w(z)(|w(z)|<1)$, such that $p_{n}(z)=L_{n}(a, b: w(z))$. Thus we have $\log p_{n}(z)=\log L_{n}(a, b: w(z))$ so that $\log p_{n}(z) \prec \log L_{n}(a, b: z)$. Hence by Lemma 3 we have the first of the inequalities.

As for the second, we also note from the above that $1 / p_{n}(z)=1 / L_{n}(a, b: w(z))$ so that $-\log p_{n}(z)=-\log L_{n}(a, b: w(z))$ and thus $-\log p_{n}(z) \prec-\log L_{n}(a, b:$ $z)$. Also $\log \left|1 / p_{n}(z)\right|$ and $\log \left|1 / L_{n}(a, b: z)\right|$ are both subharmonic in $|z|<1$ since $1 / p_{n}(z)$ and $1 / L_{n}(a, b: z)$ are analytic there. Thus by Lemma 3 again, we have the desired inequality.

\section{Proofs of Main Results}

We begin with

Proof of Theorem 1. Since $f \in T_{n}^{\alpha}[a, b], \alpha \in(0,1]$, then there exists $p_{n} \in P_{n}[a, b]$, such that $f(z)^{\alpha} / z^{\alpha}=p_{n}(z)$. Then $f^{\prime}(z)=p_{n-1}(z)(f(z) / z)^{1-\alpha}$ so that

$$
\begin{aligned}
\log \left|f^{\prime}(z)\right| & =\log \left|p_{n-1}(z)\right|+\log \left|\frac{f(z)}{z}\right|^{1-\alpha} \\
& =\log \left|p_{n-1}(z)\right|+(1-\alpha) \log \left|\frac{f(z)}{z}\right|
\end{aligned}
$$

so that, by Lemma 3,

$$
\left(\log \left|f^{\prime}(z)\right|\right)^{*}=\left(\log \left|p_{n-1}(z)\right|\right)^{*}+\left(\log \left|\frac{f(z)}{z}\right|^{1-\alpha}\right)^{*} .
$$


For $n \geq 1, f(z)$ is univalent (see [2]), so that by Lemma 2 and Corollary 1 we have

$$
\begin{aligned}
\left(\log \left|f^{\prime}(z)\right|\right)^{*} & =\left(\log \mid L_{n-1}\left(a, b: r e^{i \theta} \mid\right)^{*}+\left(\log \left|\frac{k\left(r e^{i \theta}\right)}{r}\right|^{1-\alpha}\right)^{*}\right. \\
& =\left(\log \left|\frac{L_{n-1}\left(a, b: r e^{i \theta}\right) k^{\prime}\left(r e^{i \theta}\right)^{1-\alpha}}{L_{0}\left(r e^{i \theta}\right)^{1-\alpha}}\right|\right)^{*} .
\end{aligned}
$$

Hence by Lemma 1, we have the inequality. If for some $r \in(0,1)$ and some strictly convex $\Phi$, we consider the function $f_{0}(z)$ is defined by

$$
e^{-i \alpha \gamma} \frac{f_{0}\left(z e^{i \gamma}\right)^{\alpha}}{z^{\alpha}}=L_{n}\left(a, b: z e^{i \gamma}\right)
$$

for some real $\gamma$. Then we have

$$
\begin{aligned}
e^{i \gamma(1-\alpha)} \frac{f_{0}\left(z e^{i \gamma}\right)^{\alpha-1} f_{0}^{\prime}\left(z e^{i \gamma}\right)}{z^{\alpha-1}} & =L_{n}\left(a, b: z e^{i \gamma}\right)+\frac{z e^{i \gamma} L_{n}\left(a, b: z e^{i \gamma}\right)}{\alpha} \\
& =L_{n-1}\left(a, b: z e^{i \gamma}\right)
\end{aligned}
$$

so that

$$
\left|f_{0}^{\prime}\left(z e^{i \gamma}\right)\right|=\left|L_{n-1}\left(a, b: z e^{i \gamma}\right)\right|\left|\frac{f_{0}\left(z e^{i \gamma}\right)}{z e^{i \gamma}}\right|^{1-\alpha} .
$$

Now equality in $(2)$ can be attained by taking $\left|f_{0}(z)\right|=|k(z)|$. This completes the proof.

Next we have

Proof of Theorem 2. From (3) we have

$$
\log \frac{1}{\left|f^{\prime}(z)\right|}=\log \frac{1}{\left|p_{n-1}(z)\right|}+(1-\alpha) \log \left|\frac{z}{f(z)}\right| .
$$

Hence, by Lemmas 2, 3 and Corollary 1 again, we have

$$
\begin{aligned}
\left(-\log \left|f^{\prime}(z)\right|\right)^{*} & \leq\left(\log \left|\frac{1}{L_{n-1}\left(a, b: r e^{i \theta}\right)}\right|\right)^{*}+\left(\log \left|\frac{r}{k\left(r e^{i \theta}\right)}\right|^{1-\alpha}\right)^{*} \\
& =\left(-\log \left|\frac{L_{n-1}\left(a, b: r e^{i \theta}\right) k^{\prime}\left(r e^{i \theta}\right)^{1-\alpha}}{L_{0}\left(r e^{i \theta}\right)^{1-\alpha}}\right|\right)^{*} .
\end{aligned}
$$

Hence by Lemma 1, we have the inequality. Similarly if equality is attained for some $r \in(0,1)$ and some strictly convex $\Phi$, then $f_{0}(z)$ given by $(4)$ is the equality function.

\section{Particular CASES}

With the same hypothesis as in Theorem 1 except:

(i) $n=1$, we have:

$$
\int_{-\pi}^{\pi} \Phi\left( \pm \log \left|f^{\prime}\left(r e^{i \theta}\right)\right|\right) d \theta \leq \int_{-\pi}^{\pi} \Phi\left( \pm \log \left|\frac{L_{0}\left(a, b: r e^{i \theta}\right) k^{\prime}\left(r e^{i \theta}\right)^{1-\alpha}}{L_{0}\left(r e^{i \theta}\right)^{1-\alpha}}\right|\right) d \theta .
$$


(ii) $\alpha=1$, we have:

$$
\int_{-\pi}^{\pi} \Phi\left( \pm \log \left|f^{\prime}\left(r e^{i \theta}\right)\right|\right) d \theta \leq \int_{-\pi}^{\pi} \Phi\left( \pm \log \left|L_{n-1}\left(a, b: r e^{i \theta}\right)\right|\right) d \theta .
$$

(iii) $n=\alpha=1$, we have:

$$
\int_{-\pi}^{\pi} \Phi\left( \pm \log \left|f^{\prime}\left(r e^{i \theta}\right)\right|\right) d \theta \leq \int_{-\pi}^{\pi} \Phi\left( \pm \log \left|L_{0}\left(a, b: r e^{i \theta}\right)\right|\right) d \theta .
$$

Remark 4.1. The case $n=1, a=1$ and $b=-1$ gives the estimate for the special case $s(z)=z$ of the Leung results [4].

Acknowledgements: The author acknowledges the Abdus Salam International Centre for Theoretical Physics, Trieste, Italy for providing the research paper [4].

\section{REFERENCES}

[1] K. O. Babalola and T. O. Opoola, Iterated integral transforms of Caratheodory functions and their applications to analytic and univalent functions, Tamkang J. Math., 37 (4) (Winter 2006), 355-366.

[2] K. O. Babalola, Convex null sequence technique for analytic and univalent mappings of the unit disk, Tamkang J. Math., 40 (2) (Summer 2009), 201-209.

[3] A. Baernstein, Integral means, univalent functions and circular symetrization, Acta Math., 133 (1974) 139-169.

[4] Y. J. Leung, Integral means of the derivatives of some univalent functions, Bull. London Math. Soc., 11 (1979), 289-294.

[5] G. S. Salagean, Subclasses of univalent functions, Lecture Notes in Math., 1013 (1983), 362-372. Springer-Verlag, Berlin, Heidelberg and New York.

${ }^{1}$ Department of Mathematics, University of Ilorin, P. M. B 1515, Ilorin, NigeRIA.

E-mail address: babalola.ko@unilorin.edu.ng, kobabalola@gmail.com 